amin-Antipoden zeigen auch die Transaminasebestimmungen in der Leber. Während die Konzentration der Aspartataminotransferase in der Leber durch D-Penicillamin gar nicht, durch L-Penicillamin nur geringfügig vermindert wird, ist die Konzentration der Alaninaminotransferase auch nach D-Penicillamin vermindert $(20 \%)$ und wird durch L-Penicillamin erheblich gesenkt (50\%).
Über vergleichende Untersuchungen der Wirkung der Penicillaminantipoden und ihrer Kondensationsprodukte mit Pyridoxalphosphat auf isolierte Enzyme werden wir in einer weiteren Mitteilung berichten (30).

Fräulein Brigitte Clemens ( $\dagger$ ) danken wir für ihre stets zuverlässige technische Assistenz.

\title{
Literatur
}

1. Wilson, J. E. und V. Du Vigneaud, Science (New York) 107, 635 (1948). - 2. Wilson, J. E. und V. du Vigneaud, J. biol. Chemistry 184, 63 (1950). - 3. Kuchinskas, E. J. und V. DU Vigneaud, Arch. Biochem. Biophysics 66, 1 (1957). - 4. KuchinsKas, E. J., A. Horvath und V. DU Vigneaud, Arch. Bioctem. Biophysics 68, 69 (1957) - 5. DU Vigneaud, V., E. J. KuCHINSKas und A. Horvath, Arch. Biochem. Biophysics 69, 130 (1957). 6. Asatoor, A. M., Nature (London) 203, 1382 (1964). - 7. JAFFE, I. A., K. Altman und P. Merryman, J. Clin. Invest. 43, 1869 (1964) - 8. GrbBs, K. und J. M. WALSHE, Lancet (London) I, 175 (1966). - 9. BeNESCH, R. und R. E. BeNESCH, Determination of -SH Groups in Proteins; in: Methods of Biochemical Analysis, Bd. X, S. 43. Hrsg. D. Glick, Interscience Publishers, Inc. New YorkLondon (1962); Boyer, P. D., J. Amer. chem. Soc. 76, 4331 (1954). - 10. Schlossmann, K., J. BrügGemann und F. Linen, Biochem. Z. 336, 258 (1962). - 11. Toknnies, G. und R. P. Homiller, J. Amer. chem. Soc. 64, 3054 (1942) - 12. Herken, H., G. SenFT und H. Winurzkr, Naunyn-Schmiedebergs Arch. exp. Pathol. Pharmakol. 229, 123 (1956). - 13. WACHSTEIN, M. und A. GuDartis, Amer. J. Clin. Path. 22, 652 (1952). - 14. Weber, F. und O. Wiss, Hoppe-Seylers Z. physiol. Chem. 331, 124 (1963) - 15. Hoнorst, H.-J. und M. REIM, Oxalacetat, in: Methoden der enzymatischen Analyse, Hrsg. H.-U. Bergmeyer, S. 535, Verlag Chemie GmbH, Weinheim/Bergst. (1962). - 16. Bücher, TH., R. Czok, W. Lamprecht und E. Latzko, Pyruvat, in: Methoden der enzymatischen Analyse, Hrsg. H.-U. Bergmeyer, S. 253, Verlag Chemie GmbH, Weinheim/Bergstr. (1962). - 17. Lewrs, R. E., A. L. Kunz und R. E. Bell, Laborat. Animal Care 16, 505 (1966). - 18. Persson, B. H., Acta Soc. med. Upsal. 65, 96 (1960). - 19. ThIele, V. F. und M. Brin, J. Nutrit. 90, 347 (1966). - 20. Chatterjee, A. K., S. C. Jamdar und B. B. Ghosh, Experientia, Basel 22, 794 (1966). - 21. Siegmund, P., diese Z. 1, 97 (1963). 22. Siegmund, P., F. Körber und G. Hasenaank, diese Z. 4, 307 (1966). - 23. Hedde, J. G., E. W. McHenry und G. H. Beaton, Canad. J. Biochem. 41, 1215 (1963). - 24. WACKER, A., P. Chandra und E. Heyl, Arzneimittel-Forsch., Aulendorf 16, 825 (1966). - 25. Rorzsch, W., Acta biol. med. german. 16, 329 (1966). - 26. KörnER, W. F. und H. Nowak, Int. Z. Vitaminforsch. 36, 264 (1966). - 27. Hughes, P. A. M. und D. N. Raine, Clin. chimica Acta (Amsterdam) 14, 399 (1966). - 28. Coon, W. W., Amer. J. Clin. Path. 46, 345 (1966). - 29. KöRNER, W. F. und H. NowaK, Int. Z. Vitaminforsch. 37, 89 (1967). - 30. Hasenbank, G., F. Körber und P. Siegmund, Hoppe-Seylers Z. physiol. Chem. im Druck und in Vorbereitung.

Privat-Dozent Dr. P. Siegmund 1 Berlin 33, Arnimallee 22

\section{Über die Mg-Aufnahme in Erythrocyten und Ascites-Tumorzellen}

\author{
Von H. EbeL und TH. GüNTHER \\ Aus dem Physiologisch-Chemischen Institut der Freien Universität Berlin (Direktor: Prof. Dr. Dr. E. Scbütte)
}

(Eingegangen am 20. Juli 1967)

An glykolysierenden Menschenerythrocyten, atmenden Vogelerythrocyten und glykolysierenden und atmenden Ascites-Tumorzellen wurde die $\mathrm{Mg}$-Aufnahme bei einer auf 6 bis $7 \mathrm{mMol} / \mathrm{l}$ erhöhten extrazellulären $\mathrm{Mg}$-Konzentration untersucht.

Alle untersuchten Zellen nehmen mit 1,5 mMol/l Zellen gleichviel $\mathrm{Mg}$ auf.

Die Mg-Aufnahme ist nicht durch einfache Diffusion bedingt und setzt sich aus Adsorption an die Zelloberfläche und stoffwechselabhängiger $\mathrm{Mg}$-Aufnahme in die Zelle zusammen.

Die Mg-Aufnahme wird durch Glucose und Phosphàt gefördert und durch Phlorrhizin und Arsenat gehemmt.

Die Mg-Aufnahme ist ATP-abhängig und wird durch Strophanthin-g gehemmt.

Steigende $\mathrm{Na}^{+}-\mathrm{Konzentrationen} \mathrm{fördern} \mathrm{die} \mathrm{Mg}$-Aufnahme bis zu einem Maximum bei physiologischen $\mathrm{Na}^{+}-\mathrm{Konzentrationen.} \mathrm{Bei}$ physiologischer $\mathrm{Na}^{+}$-Konzentration liegt das Maximum der die $\mathrm{Mg}$-Aufnahme fördernden $\mathrm{K}^{+}$-Konzentration bei 7,5 mMol/l; höhere $\mathrm{K}^{+}-$Konzentrationen wirken hemmend.

Es wird diskutiert, daß die membrangebundene $\mathrm{Mg}^{2+}-\mathrm{Na}^{+}-\mathrm{K}^{+}-\mathrm{AT}^{-}$Pase an der $\mathrm{Mg}-\mathrm{Aufnahme}$ beteiligt ist.

The uptake of $\mathrm{Mg}$ by glycolysing human erythrocytes, respiring bird erythrocytes, and glycolysing and respiring Ascites tumor cells was studied at increased levels $(6-7 \mathrm{mM})$ of extracellular $\mathrm{Mg}$.

In all the cells, the uptake of $\mathrm{Mg}$ was the same, i. e., $1.5 \mathrm{mmoles} / \mathrm{l}$ cells.

The uptake of $\mathrm{Mg}$ is not the result of simple diffusion; it is due to the combined effects of adsorption onto the cell surface and the metabolic-dependent uptake of $\mathrm{Mg}$ into the cell.

The uptake of $\mathrm{Mg}$ is promoted by glucose and phosphate and inhibited by phlorrhizin and arsenate.

The uptake of $\mathrm{Mg}$ is ATP-dependent and is inhibited by strophanthin-g.

Increasing concentrations of $\mathrm{Na}^{+}$promote the uptake of $\mathrm{Mg}$ to a maximum corresponding to the physiological concentration of $\mathrm{Na}^{+}$. At physiological concentrations of $\mathrm{Na}^{+}$, the optimum concentration of $\mathrm{K}^{+}$for the promotion of $\mathrm{Mg}$-uptake was $7.5 \mathrm{mM}$; higher concentrations of $\mathrm{K}^{+}$were inhibitory.

It is suggested that the membrane-bound $\mathrm{Mg}^{2+}-\mathrm{Na}^{+}-\mathrm{K}^{+}-\mathrm{ATPase}$ is involved in the uptake of $\mathrm{Mg}$. 
Aus Versuchen am Ganztier ist bekannt, daß Zellmembranen für $\mathrm{Mg}$ permeabel sind: Der $\mathrm{Mg}$-Bestand von Leber, Niere, Herzmuskel und Tumorzellen wird innerhalb von drei Stunden vollständig gegen intraperitoneal injiziertes ${ }^{28} \mathrm{Mg}$ ausgetauscht $(1,2)$. Durch Permeabilitätsmessungen allein kann jedoch nicht entschieden werden, ob $\mathrm{Mg}$ passiv oder aktiv aufgenommen wird. Dies läßt sich erst beurteilen, wenn die elektrische Potentialdifferenz der betreffenden Zelle und ihre intrazelluläre Mg-Ionenkonzentration bekannt sind. Bei Ascites-Tumorzellen gelang es, eine elektrische Potentialdifferenz von $-11,1 \mathrm{mV}$ zu messen (3). Für die intrazelluläre $\mathrm{Mg}$-Ionenkonzentration wurde ein Wert von $1,6 \mathrm{mMol} / \mathrm{l}$ ermittelt (4). $\mathrm{Da}$ die extrazelluläre $\mathrm{Mg}$ Ionenkonzentration nur etwa $0,6 \mathrm{mMol} / l$ beträgt, $\mathrm{mu} \beta$ es sich bei der Mg-Aufnahme in Mg-reiche Zellen, z. B. Ascites-Tumorzellen, aus thermodynamischen Gründen um einen aktiven Transport handeln.

Weil aktive Transportleistungen energieabhängig sind, haben wir in der vorliegenden Arbeit mit verschiedenen Stoffwechselgiften den Einfluß des Stoffwechsels auf die Mg-Aufnahme bei drei verschiedenen Zelltypen untersucht. Wir verwendeten Erythrocyten vom Menschen als rein glykolysierende, Hühnererythrocyten als nur atmende und Ascites-Tumorzellen als atmende und glykolysierende Zellen. Zur weiteren Charakterisierung prüften wir außerdem den Einfluß von Strophanthin-g und verschiedenen extrazellulären Ionen auf die $\mathrm{Mg}$ Aufnahme.

\section{Methodik \\ Prinzip}

Die Zellen wurden in Ringerlösung, deren Mg-Konzentration bei den Versuchen mit Erythrocyten auf $7 \mathrm{mMol} / l$ und bei den Versuchen mit Ascites-Tumorzellen auf $6 \mathrm{mMol} / l$ erhöht war, inkubiert. Aus der Abnahme der extrazellulären $\mathrm{Mg}$-Konzentration während der Inkubation wurde die $\mathrm{Mg}$-Aufnahme in die Zelle für dieselbe Zeit berechnet und als $\Delta \mathrm{Mg}^{2+}(\mathrm{mMol} / /$ Zellen) angegeben. Die Zellen wurden jeweils solange inkubiert, bis die extrazelluläre Mg-Konzentration auf einen konstanten Wert abgesunken war: Die Inkubationszeit betrug bei Humanerythrocyten und AscitesTumorzellen 60 Min., bei Hühnererythrocyten 90 Min. (Abb. 1)

\section{Gewinnung der Zellen}

Blut wurde aus den Armvenen gesunder männlicher Versuchspersonen oder aus den Flügelvenen erwachsener Hähne in heparinisierte Gläser abgelassen und sofort $5 \mathrm{Min}$. bei $3000 \mathrm{~g}$ zentrifugiert. Serum und Leukocyten wurden abgesaugt.

Ehrlich Ascites-Tumorzellen (ELD) wurden 7-9 Tage nach intraperitonealer Inokulation durch Punktion der Bauchhöhle gewonnen und zur Vermeidung mechanischer Schädigung bei $600 \mathrm{~g}$ zentrifugiert. Mit zwei- bis dreimaligem Waschen wurden die Blutbeimengungen aus den Ascites-Tumorzellen entfernt.

\section{Inkubation der Zellen}

Die Zellen wurden in Mg-freier Ringerlösung mit einem Glucosegehalt von $195 \mathrm{mg} / 100 \mathrm{ml}$ in Warburg-Gefäßen suspendiert. Die Zellkonzentrationen betrugen bei Erythrocyten etwa 25\% (v/v) und bei Ascites-Tumorzellen etwa 12\% (v/v). Die Gefäße wurden bei $38^{\circ}$ mit Luft geschüttelt. Die Frequenz betrug bei Versuchen mit Erythrocyten 120/Min., bei Versuchen mit Ascites-Tumorzellen 160/Min. Die Amplitude betrug $2 \mathrm{~cm}$. Zum Temperaturausgleich wurden die Zellen $15 \mathrm{Min}$. bei $38^{\circ}$ vorinkubiert. Anschließend wurde die $\mathrm{Mg}$-Aufnahme durch Zugabe von $\mathrm{MgCl}_{2}$ Lösung gestartet. Sofort nach Versuchsbeginn und am Ende der Inkubation wurde ein aliquoter Teil der Zellsuspension entnommen und 5 Min. bei $3000 \mathrm{~g}$ zentrifugiert. Im Zellsediment wurde das extrazelluläre Flüssigkeitsvolumen und im Überstand die $\mathrm{Mg}$ Konzentration bestimmt.

\section{Bestimmung der $E Z F$}

Die extrazelluläre Flüssigkeit (EZF) zwischen den sedimentierten Zellen wurde mit Rohrzucker nach WAHLER bestimmt (5). Sie betrug $15 \%(v / v)$ bei Erythrocyten und $18 \%(v / v)$ bei Ascites-Tumorzellen.

\section{Mg-Bestimmung}

Zur Mg-Bestimmung wurde der Uberstand 1:7 mit bidest. Wasser verdünnt und im Verhältnis 1:11 mit $10 \mathrm{proz}$. (w/v) Trichloressigsäure enteiweißt. Im klaren Überstand wurde $\mathrm{Mg}$ mit der Titangelbmethode von OrANGE und Rhern (6) bei $546 \mathrm{~nm}$ in Küvetten mit $5 \mathrm{~cm}$ Schichtdicke im Eppendorf Photometer bestimmt. Die Standardabweichung der Methode war bei Vierfachbestimmungen $\pm 3 \%$.

\section{Ergebnisse}

\section{Zeitabbängigkeit der $M g$-Aufnabme}

Erythrocyten und Ascites-Tumorzellen nehmen bis zur Einstellung eines neuen Gleichgewichtes etwa gleichviel $\mathrm{Mg}$ auf (Abb. 1, Tab. 1). Für die maximale $\mathrm{Mg}$-Aufnahme benötigen Erythrocyten vom Menschen 50 Min., Hühnererythrocyten $90 \mathrm{Min}$. und Ascites-Tumorzellen $60 \mathrm{Min}$. Die Menge des aufgenommenen Mg ist also unabhängig von der intrazellulären $\mathrm{Mg}$-Konzentration (Summe des komplex gebundenen und ionisierten $\mathrm{Mg}$ ).

Tab. 1

Geschwindigkeit und Größe der maximalen Mg-Aufnahme verschiedener Zellen. Es sind $\mathrm{Mg}_{\mathrm{i}}=$ intrazelluläre $\mathrm{Mg}$-Konzentration (eigene Messung), $\mathbf{M g}_{\mathrm{e}}=$ extrazelluläre $\mathrm{Mg}$-Konzentration, $\Delta \mathrm{Mg}^{2+}$ $=M g$-Aufnahme, $t_{m}=$ Zeit bis zum Erreichen der maximalen $M g-$ Aufnahme

\begin{tabular}{lcccc}
\hline Zellart & $\begin{array}{c}\mathrm{Mg}_{\mathrm{i}} \\
(\mathrm{mMol} / \mathrm{l})\end{array}$ & $\begin{array}{c}\mathrm{Mg}_{\mathrm{e}} \\
(\mathrm{mMol} / \mathrm{l})\end{array}$ & $\begin{array}{c}\Delta \mathrm{Mg}^{2+} \\
(\mathrm{mMol} / l \text { Zellen })\end{array}$ & $\begin{array}{c}\mathrm{t}_{\mathrm{m}} \\
(\mathrm{Min} .)\end{array}$ \\
\hline Menschenerythrocyten & 2,4 & 7 & $\left.1,44 \pm 0,18^{*}\right)$ & 50 \\
Hühnererythrocyten & 8,1 & 7 & $1,52 \pm 0,13$ & 90 \\
Ascites-Tumorzellen & 8,1 & 6 & $1,56 \pm 0,15$ & 60 \\
\hline
\end{tabular}

*) mittlerer Fehler des Mittelwertes

\section{Mg-Aufnabme als Funktion der extrazellulären Mg-Konzen- tration}

In einer weiteren Versuchsserie haben wir an Hühnererythrocyten die Mg-Aufnahme als Funktion der extrazellulären $\mathrm{Mg}$-Konzentration untersucht. Hierzu wurden die Zellen nach Waschen in Mg-freier Ringerlösung mit zunehmenden $\mathrm{Mg}-$ Konzentrationen inkubiert. Wir fanden, daß die Mg-Aufnahme asymptotisch einem Maximalwert zustrebt, der etwa bei $20 \mathrm{mMol} \mathrm{Mg} / l$ erreicht wird. Daraus folgt, daß die $\mathrm{Mg}$-Aufnahme keine reine Diffusion sein kann (Abb. 2).

\section{Mg-Aufnabme in Abbängigkeit vom Stoffwechsel}

Es wurde zunächst geprüft, ob die Mg-Aufnahme überwiegend von einer physikalischen oder chemischen Reaktion abhängig ist. Hierzu untersuchten wir die $\mathrm{Mg}$ Aufnahme bei verschiedenen Temperaturen. Wir fanden, $\mathrm{da} \beta$ die $\mathrm{Mg}$-Aufnahme mit ansteigender Temperatur bis zu einem optimalen Wert bei $38^{\circ}$ zunimmt und bei höherer Temperatur wieder abnimmt .(Tab. 2). Für die Mg-Aufnahme zwischen $28^{\circ}$ und $38^{\circ}$ berechneten wir einen Temperaturkoeffizienten von $Q_{10}=1,38$ und eine Aktivierungsenergie von $5,7 \mathrm{kcal} \cdot \mathrm{Mol}^{-1}$. 


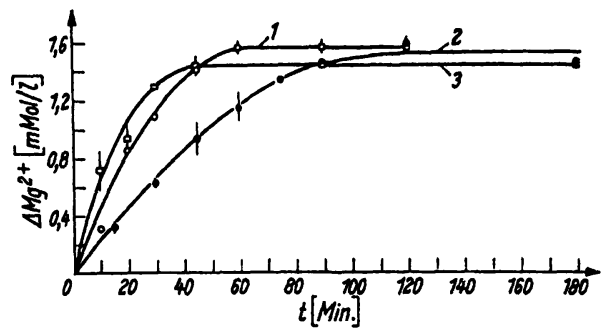

Abb. 1

Zeitlicher Verlauf der Mg-Aufnahme. $1=$ Ascites-Tumorzellen, Zellkonzentration $12 \%(v / v), 2=$ Hühnererythrocyten, Zellkonzentration $25 \%(v / v), 3=$ Menschenerythrocyten, Zellkonzentration $29 \%$ (v/v). $195 \mathrm{mg} / 100 \mathrm{ml}$ Glucose suspendiert Die $\mathrm{Mg}$ Konzentration betruge suspendiert. Die $\mathrm{Mg}$ Konzentration betrug 7 mMol/l bei Erythrocyten, 6 mMol $/ l$ bei Ascites-Tumorzellen. Die Wasserbadtemperatur betrug $38^{\circ}$, die SchütAscites-Tumorzellen 160/Min., die Amplitude $2 \mathrm{~cm}$

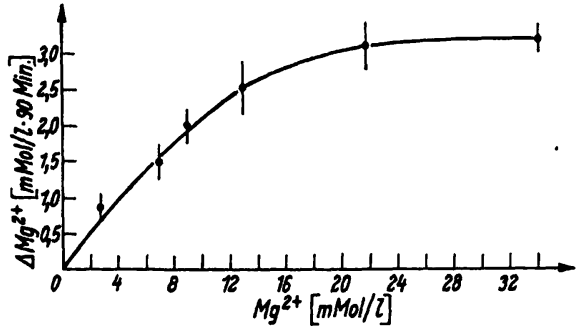

Abb. 2

Mg-Aufnahme von Hühnererythrocyten als Funktion der extrazellulären $\mathrm{Mg}$ Konzentration. Die Zellen wurden vor dem Versuch einmal in Mg-freier Ringerlösung gewaschen

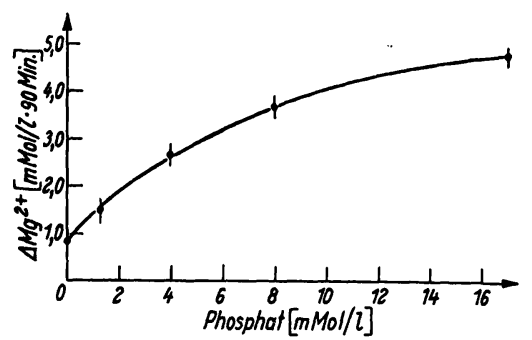

Abb. 3

Mg-Aufnahme von Hühnererythrocyten in Abhängigkeit von der Phosphatkonzentration. Die Zellen wurden vor dem Versuch einmal in Phosphat- und $\mathrm{Mg}$-freier Ringerlösung gewaschen
Für die Mg-Aufnahme von Hefezellen ist die Anwesenheit von Glucose in der extrazellulären Flüssigkeit erforderlich $(7,8)$. Wir untersuchten daher, ob dies auch für die Mg-Aufnahme von Erythrocyten und AscitesTumorzellen gilt, indem wir den Glucosetransport dieser

Tab. 2

Mg-Aufnahme von Hühnerythrocyten bei verschiedenen Temperaturen. Die Mg-Aufnahme wurde nach $10 \mathrm{Min}$. Temperaturausgleich mit $\mathrm{MgCl}_{2}$-Lösung gestartet. Die $\mathrm{Mg}$-Aufnahme bei $38^{\circ}$ wurde gleich $100 \%$ gesetzt

\begin{tabular}{ccr}
\hline Temperatur & $\Delta \mathrm{Mg}^{2+}(\mathrm{mMol} / \mathrm{l} \cdot 90 \mathrm{Min})$. & $\%$ \\
\hline $0^{\circ}$ & $0,65 \pm 0,04$ & 43 \\
$28^{\circ}$ & $1,10 \pm 0,03$ & 72 \\
$38^{\circ}$ & $1,52 \pm 0,13$ & 100 \\
$48^{\circ}$ & $0,66 \pm 0,04$ & 42 \\
\hline
\end{tabular}

Zellen mitPhlorrhizin hemmten. Wir fanden, daß unter diesen Bedingungen die Mg-Aufnahme aller drei Zelltypen auf die Hälfte des Kontrollwertes absinkt (Tab. 3). $\mathrm{Da}$ Glucose nur nach Phosphorylierung in der Zelle umgesetzt wird, prüften wir den Einfluß von Phosphat (in Gegenwart von Glucose) auf die Mg-Aufnahme von Hühnererythrocyten. Es ergab sich, daß mit zunehmender extrazellulärer Phosphatkonzentration und zugleich vermehrtem Phosphattransport in die Zelle auch zunehmende Mengen von $\mathrm{Mg}$ aufgenommen werden (Abb. 3).

Die Abhängigkeit der Mg-Aufnahme von der Phosphatkonzentration ließ sich noch zusätzlich durch ihre kompetetive Hemmbarkeit mittels Arsenat zeigen. Wie aus Tabelle 3 hervorgeht, wird bei Erythrocyten und As-
cites-Tumorzellen die Mg-Aufnahme in Gegenwart von Phosphat durch Arsenat um 50\% gehemmt.

Die weitere Analyse der an der Mg-Aufnahme beteiligten chemischen Reaktionen gelang mit Stoffwechselinhibitoren. Wird die Glykolyse durch Zusatz von Monojodacetat gehemmt, wobei z. B. der ATPGehalt in Menschenerythrocyten um $90 \%$ abnimmt (9), so sinkt die $\mathrm{Mg}$-Aufnahme von Hühnererythrocyten und Ascites-Tumorzellen auf etwa $60 \%$ der Kontrolle ab. Bei den atmenden Hühnererythrocyten ist die Unterbrechung der Glykolyse ohne Wirkung (Tab. 3).

Hemmten wir dagegen die Atmung durch Blausäure, was z. B. bei Taubenerythrocyten eine Abnahme des ATP um etwa $80 \%$ zur Folge hat (9), so sinkt nur bei Hühnererythrocyten und Ascites-Tumorzellen die Menge des aufgenommenen $\mathrm{Mg}$, während man an den gärenden Menschenerythrocyten keinen Effekt feststellen kann (Tab. 3).

Die Wirkung der Atmung ließ sich durch die Entkopplung der Atmungskettenphosporylierung mit 2,4Dinitrophenol weiter differenzieren. Wir fanden, da $B$ bei atmenden Hühnererythrocyten und Ascites-Tumorzellen die $\mathrm{Mg}$-Aufnahme durch Entkopplung auf die Hälfte des Kontrollwertes gesenkt wird (Tab. 3).

\section{Einfluß von Stropbantbin-g auf die $M g$-Aufnabme}

Der aktive $\mathrm{Na}^{+}$- und $\mathrm{K}^{+}$-Transport an tierischen Zellen wird durch Strophanthin-g gehemmt; wir prüften deshalb den Einfluß von Strophanthin-g auf die Mg-Aufnahme. Wir fanden, daß Strophanthin-g in einer Kon-

Tab. 3

Wirkung verschiedener Stoffwechselgifte auf die $\mathbf{M g}=$ Aufnahme. Die Zellen wurden 15 Min. mit dem jeweiligen Inhibitor vorinkubiert

\begin{tabular}{|c|c|c|c|c|c|c|}
\hline Inhibitor & $\begin{array}{l}\text { Humanerythro } \\
\Delta M g^{2+} \\
\text { (mMol } / l \cdot 60 \text { Min.) }\end{array}$ & $(\%)$ & $\begin{array}{c}\text { Hühnererythro } \\
\Delta \mathrm{Mg}^{2+} \\
\text { (mMol } / \mathrm{l} \cdot 90 \mathrm{Min} .)\end{array}$ & $(\%)$ & $\begin{array}{c}\text { Ascites-Tumorzellen } \\
\qquad \mathrm{Mg}^{2+} \\
\text { (mMol } / l \cdot 60 \mathrm{Min} .)\end{array}$ & (\%) \\
\hline \multirow[t]{2}{*}{ 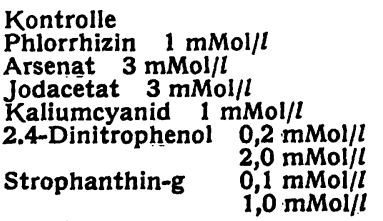 } & $\begin{array}{l}1,44 \pm 0,18 \\
0,65 \pm 0,03 \\
0,78 \pm 0,08 \\
0,82 \pm 0,16 \\
1,44 \pm 0,02 \\
1,44 \pm 0,18 \\
1,48 \pm 0,04\end{array}$ & $\begin{array}{r}100 \\
45 \\
54 \\
57 \\
100 \\
100 \\
103\end{array}$ & $\begin{array}{l}1,52 \pm 0,13 \\
0,70 \pm 0,01 \\
0,79 \pm 0,01 \\
1,57 \pm 0,12 \\
0,72 \pm 0,02 \\
1,28 \pm 0,10 \\
0,93 \pm 0,00\end{array}$ & $\begin{array}{r}100 \\
46 \\
52 \\
103 \\
47 \\
83 \\
60\end{array}$ & $\begin{array}{l}1,56 \pm 0,15 \\
0,83 \pm 0,02 \\
0,65 \pm 0,01 \\
0,92 \pm 0,05 \\
0,97 \pm 0,01 \\
1,39 \pm 0,05 \\
0,78 \pm 0,00\end{array}$ & $\begin{array}{r}100 \\
53 \\
42 \\
59 \\
62 \\
89 \\
55\end{array}$ \\
\hline & $0,7.1 \pm 0,04$ & & $0,80 \overline{ \pm} 0,03$ & 53 & $0,74 \pm 0,02$ & 48 \\
\hline
\end{tabular}




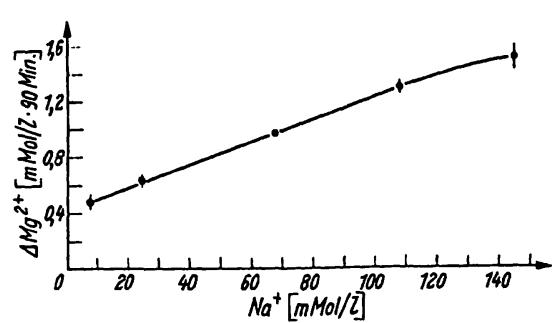

Abb. 4

Mg-Aufnahme von Hühnererythrocyten in Abhängigkeit von der extrazellulären $\mathrm{Na}^{+}-$Konzentration. Die Zellen wurden vor dem Versuch in $\mathrm{Na}^{+}$- und $\mathrm{Mg}$-freier Ringetlösung gewaschen. Das $\mathrm{NaCl}$ wurde beim Waschen und im Versuch durch isoosmolare Konzentration von Cholinchlorid ersetzt. Die $\mathrm{K}^{+}$-Konzentration betrug jeweils $7,5 \mathrm{mMol} / \mathrm{l}$

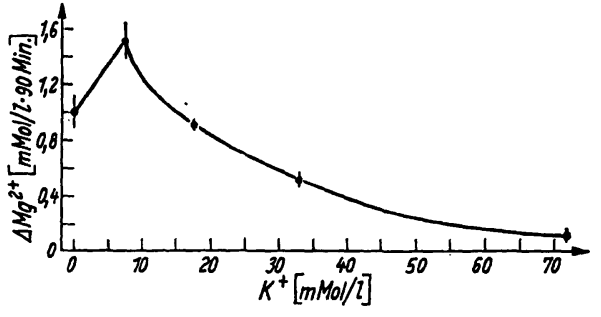

Abb. 5;

Mg-Aufnahme von Hühnererythrocyten in Abhängigkeit von der extrazellulären $\mathrm{K}^{+}$Konzentration. Die Zellen wurden vor dem Versuch einmal in $\mathrm{K}^{+}$- und $\mathrm{Mg}$-freier Ringetlösung gewaschen. Die $\mathrm{Na}^{+}-$Konzentration betrug jeweils $140 \mathrm{mMol} / \mathrm{l}$

bleibende $\mathrm{Mg}$-Aufnahme durch Diffusion erklären. Gegen diese Annahme spricht aber, daß die Mg-Aufnahme mit steigenden extrazellulären $\mathrm{Mg}$-Ionenkonzentrationen nicht linear zunimmt. Sie ähnelt vielmehr einer LANGMUIRschen Adsorptionsisotherme mit einer Sättigung bei $20 \mathrm{mMol} / \mathrm{Mg}$. Eine entsprechende Beziehung wurde auch für die stoffwechselabhängige $\mathrm{Mg}$-Aufnahme in Hefezellen (8) und für die transzelluläre $\mathrm{Mg}$-Aufnahme entlang dem elektrochemischen Gradienten im Darm von Ratten (10) und Schafen (11) gefunden. Die einge-

zentration von $0,1-1,0 \mathrm{mMol} / \mathrm{l}$ die $\mathrm{Mg}$-Aufnahme der Erythrocyten und Ascites-Tumorzellen auf die Hälfte des Kontrollwertes senkt (Tab. 3).

Mg-Aufnabme als Funktion der extrazellulären $\mathrm{Na}^{+}$und $K^{+}$-Konzentration

Bei tierischen Zellen ist der aktive $\mathrm{Na}^{+}$- und $\mathrm{K}+$ Transport unter anderem von der extrazellulären $\mathrm{Na}^{+}$und $\mathrm{K}^{+}$-Konzentration abhängig. Daher untersuchten wir bei Hühnererythrocyten den Einfluß der extrazellulären $\mathrm{Na}^{+}$- und $\mathrm{K}^{+}-$Konzentration auf die $\mathrm{Mg}$-Aufnahme.

Wir fanden, daß mit zunehmender extrazellulärer $\mathrm{Na}+$ Konzentration bei konstanter $\mathrm{K}^{+}-$Konzentration die $\mathrm{Mg}$-Aufnahme vergrößert wird. Maximale Mg-Aufnahme erfolgt bei physiologischer extrazellulärer $\mathrm{Na}{ }_{-}$ Konzentration, halbmaximale bei etwa $50 \mathrm{mMol} / \mathrm{l}$ (Abb. 4).

Bei steigender $\mathrm{K}^{+}-$Konzentration und physiologischer $\mathrm{Na}+-$ Konzentration "nimmt die Mg-Aufnahme bis zu einem Optimum bei $7,5 \mathrm{mMol} / l \mathrm{~K}+\mathrm{zu}$. Durch höhere $\mathrm{K}^{+}$-Konzentrationen witd die $\mathrm{Mg}$-Aufnahme zunehmend gehemmt (Abb. 5).

\section{Diskussion}

Aus den Experimenten folgt, da $\beta$ die Mg-Aufnahme in Erythrocyten und Ascites-Tumorzellen bei erhöhten extrazellulären $\mathrm{Mg}$-Konzentrationen aus einem stoffwechselunabhängigen und einem stoffwechselabhängigen Teil besteht. Die Mg-Aufnahme ist infolge der gewählten hohen extrazellulären $\mathrm{Mg}$-Konzentration bergab getichtet. Unter unseren Versuchsbedingungen beträgt der Quotient aus extra- und intrazellulärer Mg-Ionenkonzentration z. B. bei Ascites-Tumorzellen 3, 75.

Den stoffwechselunabhängigen Anteil der Mg-Aufnahme mißt man, wenn der Zellstoffwechsel durch Abkühlen auf $0^{\circ}$ verlangsamt oder durch Gifte gehemmt wird. Unter diesen Bedingungen sinkt die Mg-Aufnahme von Erythrocyten und Ascites-Tumorzellen auf die Hälfte ab. Wegen der einwärts gerichteten Gradienten der $\mathrm{Mg}$-Ionenkonzentrationen könnte man die ver- hende Analyse der Mg-Aufnahme von Hefezellen ergab bei höheren $\mathrm{Mg}$-Konzentrationen keine Übereinstimmung mit der Langmurrschen Formel. Die Aufnahme ließ sich besser beschreiben, wenn sie aus der Summe zweier Langmuir-Gleichungen mit zwei verschiedenen Gleichgewichtskonstanten berechnet wurde (8). $\mathrm{Da}$ die Langmuir-Gleichung und die MichaelisMenten-Gleichung formal gleich sind, weil beide einen Vorgang beschreiben, bei dem unter unseren Bedingungen ein membrangebundener Reaktionspartner limitierend wirkt (12), könnte man sich die Aufnahmekurven von Hefezellen und Erythrocyten aus einer Adsorptionsisothermen und einer stoffwechselabhängigen Michaelis-Menten-Kinetik zusammengesetzt vorstellen. Für die Bindung kämen vor allem Carboxyl- und Phosphatgruppen in Frage, wie es für die $\mathrm{Mn}$ - und $\mathrm{Mg}$-Adsorption an Hefezellen angegeben wird (13).

Die bergab verlaufende Mg-Aufnahme ist energieabhängig. Wird die Glucoseaufnahme mit Phlorrhizin gehemmt, dann sinkt die $\mathrm{Mg}$-Aufnahme von Erythrocyten und Ascites-Tumorzellen auf die Größe der stoffwechselunabhängigen Adsorption ab. Auch die Hemmung der Phosphataufnahme bzw. die Hemmung einer phosphorylierenden Reaktion mit Hilfe von Arsenat hat eine Abnahme der Mg-Aufnahme bei allen drei Zelltypen zur Folge. Dieses Verhalten ist auch von Hefezellen bekannt, bei denen ein Teil der $\mathrm{Mg}$-Aufnahme an die gleichzeitige Aufnahme von Glucose und Phosphat gebunden ist $(7,8)$. Auch bei isolierten intakten Mitochondrien ist die stoffwechselabhängige $\mathrm{Mg}$-Aufnahme maximal, wenn gleichzeitig Phosphat aufgenommen wird (14).

Die ATP-Abhängigkeit der Mg-Aufnahme ergibt sich aus folgenden Versuchen: Hemmt man die Glykolyse von Menschenerythrocyten und Ascites-Tumorzellen durch Monojodacetat, dann wird kein $\mathrm{Mg}$ mehr aufgenommen. Das gleiche hat eine Hemmung der Atmung mit Cyanid und die Entkopplung der oxydativen Phosphorylierung mit 2.4Dinitrophenol bei Hühnerexythrocyten zur Folge. Hiermit stimmt überein, daß der transzelluläre ${ }^{28} \mathrm{Mg}$ influx am Meerschweinchendarm durch Cyanid und Jodacetat gesenkt, der efflux hingegen 
gesteigert wird (15). Isolierte atmende Mitochondrien beziehen die Energie für die aktive $\mathrm{Mg}$-Aufnahme ebenfalls vom ATP oder vom Elektronentransportsystem (16).

Aus diesen Untersuchungen kann über die Art der Kopplung zwischen $\mathrm{Mg}$-Aufnahme und intrazellulärer ATP-Konzentration nichts ausgesagt werden. Es ist möglich, daß die $\mathrm{Mg}$-Aufnahme von einer ATP-benötigenden enzymatischen Reaktion abhängig ist. Es ist aber nicht auszuschließen, daß mit der starken Abnahme der intrazellulären ATP-Konzentration unter der Einwirkung der Stoffwechselgifte eine Zunahme des ionisierten $\mathrm{Mg}^{2+}$ verbunden isț, wenn das vorher an ATP komplex gebundene $\mathrm{Mg}$ nicht vollständig von anderen intrazellulären Komplexbildern gebunden wird. Dadurch würde der einwärts gerichtete $\mathrm{Mg}$-Ionengradient abnehmen und die $\mathrm{Mg}$-Aufnahme geringer werden.

Die Mg-Aufnahme von Erythrocyten und AscitesTumorzellen wird durch Strophanthin-g, das auch andere aktive Transporte ohne Verminderung der ATPKonzentration blockiert $(17,18)$, gehemmt. Hieraus kann man ableiten, daß $\mathrm{Mg}$ über einen durch Strophanthin hemmbaren Trägermechanismus aufgenommen wird, dessen Energiebedarf von ATP oder einem Zwischenprodukt der oxydativen Phosphorylierung gedeckt wird. Über den genauen Angriffspunkt von Strophanthin-g läßt sich nichts sagen. Es kann sich dabei um eine direkte Wirkung auf den $\mathrm{Mg}$-Trägermechanismus handeln. Es wäre aber auch möglich, daß der $\mathrm{Mg}$ Transport mit dem $\mathrm{Na}^{+}-$Transport gekoppelt ist. Eine derartige Beziehung wurde auch für den transzellulären Mg-Transport im Darm angenommen (19). Strophanthin-g könnte demnach den $\mathrm{Mg}$-Transport hemmen, indem es den aktiven $\mathrm{Na}+$-Transport unterbindet. Im letzten Fall würden somit gleiche Bedingungen wrie beim aktiven Glucosetransport im Darm herrschen, wo die
Hemmung des Glucosetransports durch Strophanthin-g durch eine Hemmung des $\mathrm{Na}^{+}-$Transports erklärt wird (20).

Die Erhöhung des $\mathrm{Mg}$-Transports durch $\mathrm{Na}^{+}$und $\mathrm{K}+$ erfolgt bei $\mathrm{Na}^{+}$- und $\mathrm{K}+-$ Konzentrationen, die auch die $\mathrm{Mg}^{2+} \mathrm{Na}^{+} \mathrm{K} \mathrm{K}_{-} \mathrm{ATP}$ ase der Zellmembran stimulieren. Die membrangebundene ATPase der menschlichen Erythrocyten ist bei einer $\mathrm{K}^{+}-$Konzentration von $20 \mathrm{mMol} / \mathrm{l}$ und einer $\mathrm{Na}+-$ Konzentration von $120 \mathrm{mMol} / \mathrm{l}$ maximal aktiviert (21). Bei Hühnererythrocyten ist die $\mathrm{Mg}$-Aufnahme bei einer $\mathrm{K}^{+}$-Konzentration von 7,5 $\mathrm{mMol} / l$ und einer $\mathrm{Na}+-$ Konzentration von $150 \mathrm{mMol} / \mathrm{l}$ maximal. Beide Systeme sind durch Strophanthin-g hemmbar. Die membrangebundene $\mathrm{Mg}^{2+}-\mathrm{Na}_{-}-\mathrm{K}+_{-}$ ATPase menschlicher Erythrocyten ist bei $0,6 \mathrm{mMol} / \mathrm{l}$ Strophanthin-g vollständig gehemmt (21). Bei menschlichen Erythrocyten wird die Mg-Aufnahme durch 0,13 $\mathrm{mMol} / l$ Strophanthin-g vollständig gehemmt.

Aus diesen Übereinstimmungen kann man schließen, dal3 die membrangebundene $\mathrm{Mg}^{2+}-\mathrm{Na}+-\mathrm{K}+-\mathrm{ATPase}$ auch an der $\mathrm{Mg}$-Aufnahme beteiligt ist. Legt man das von SKou entwickelte ATP-ase Modell $(22,23)$ für den aktiven $\mathrm{Na}^{+}$- und $\mathrm{K}^{+}-$Transport zugrunde, so könnte $\mathrm{K}$

$\mathrm{Mg}$ außen in den $\underset{\mathrm{Ea}}{\mathrm{E}} \mathrm{Mg}_{2}$ ATP Komplex eintreten.

Wenn beim Transportvorgang ATP zu ADP und Phosphat gespalten wird und sich dieser Komplex über $\longrightarrow \underset{\mathrm{Na}}{\mathrm{E}} \sim \mathrm{P}+2 \mathrm{Mg}+\mathrm{ADP} z \underset{\mathrm{K}}{\mathrm{K}}+\mathrm{P}_{\mathrm{i}}$ umwandelt, könnte $\mathrm{Mg}^{2+}$ innen freigesetzt werden, denn die Stabilitätskonstante des sich auf der Innenseite ergebenden $\mathrm{Mg}$-Chelates ist kleiner. Über dieses System könnte auch unter in-vivo-Bedingungen mit niedrigeren extrazellulären $\mathrm{Mg}$-Ionenkonzentrationen $\mathrm{Mg}$ aktiv aufgenommen werden.

\section{Literatur}

1. Rogers, T. A. und P. E. Mahan, Proc. Soc. exp. Biol. Med. 100, 235 (1959). - 2. Rogers, T. A., F. L. Havien und P. E. Mafian, J. Nat. Cancer Inst., Wash. 25, 887 (1960). - 3. Hempling, H. G., J. Cellul. Comp. Physiol., Philadelphia 60, 181 (1962). - 4. GüNTher, TH., Z. Naturforsch. 22b, 149 (1967). - 5. WaHLER, B. E., Hoppe-Seyler's Z. physiol. Chem. 306, 84 (1956). - 6. Orange, M. und H. C. Rhein, J. biol. Chemistry 189, 379 (1951). - 7. Rothstein, A., A. Hayes, D. Jennings und D. Hooper, J. gen. Physiol. 41, 585 (1958). - 8. Netrer, H. und L. SACris, Biochem. Z. 334, 18 (1961). - 9. GerLACH, E. und K. LüBBEN, Pflügers Arch. ges. Physiol. Menschen Tiere 269, 520 (1959). 10. Ross, D. B., J. Physiology 160, 417 (1962). - 11. CARE, A. D. und A. Th. van't Krooster, J. Physiology 177, 174 (1965). 12. Aschнerm, E., Experientia (Basel) 16, 305 (1960). - 13.
Rothstein, A. und A. D. Hayes, Arch. Biochem. Biophysics 63, 87 (1956). - 14. Carafoli, E., C. S. Rossi und A. L. Lehninger, J. biol. Chemistry 239, 3055 (1964). - 15. Ross, D. B. und A. D. Care, Biochem. J. 82, 21 P (1962). - 16. Bierley, G. B., E. Murer und D. E. GreEN, Science (Washington) 140, 60 (1963). - 17. SchatzmanN, H. J., Helv. physiol. pharmakol. Acta 11, 346 (1956). - 18. Whittam, R., J. Physiology 140, 479 (1958). - 19. Ross, D. B., Nature (London) 189, 840 (1961). - 20. Csákx, T. Z. und Y. Hara, Amer. J. Physiol. 209, 467 (1965). - 21. Post, R. L., C. R. Meritt, C. R. Kinsolving und C. D. Albright, J. biol. Chemistry 235, 1796 (1960). - 22. Skou, J., Biochim. biophysica Acta (Amsterdam) 42, 6 (1960). - 23. Skou, J., in: Progress in biophysics and molecular biology, Edt. J. A. V. Butler und H. E. Huxley Bd. 14, S. 131, Pergamon Press, Oxford (1964).

Priv.-Doz. Dr. Th. Günther 1 Berlin 33, Arnimallee 22 